\title{
Urgences
}

\section{Les images de la fertilité au Pérou}

\section{Bill Vazan}

Numéro 17-18, octobre 1987

L'esprit des lieux

URI : https://id.erudit.org/iderudit/025431ar

DOI : https://doi.org/10.7202/025431ar

Aller au sommaire du numéro

Éditeur(s)

Urgences

ISSN

0226-9554 (imprimé)

1927-3924 (numérique)

Découvrir la revue

Citer ce document

Vazan, B. (1987). Les images de la fertilité au Pérou. Urgences, (17-18), 169-178.

https://doi.org/10.7202/025431ar

Ce document est protégé par la loi sur le droit d'auteur. L’utilisation des services d'Érudit (y compris la reproduction) est assujettie à sa politique d'utilisation que vous pouvez consulter en ligne.

https://apropos.erudit.org/fr/usagers/politique-dutilisation/
Cet article est diffusé et préservé par Érudit.

Érudit est un consortium interuniversitaire sans but lucratif composé de l’Université de Montréal, l'Université Laval et l'Université du Québec à Montréal. Il a pour mission la promotion et la valorisation de la recherche. https://www.erudit.org/fr/ 


\section{Bill Vazan \\ LES IMAGES DE LA FERTILITÉ AU PÉROU}

La surface de la terre porte la trace de symboles à grande échelle laissés par de nombreuses sociétés préhistoriques. Réalistes, abstraits ou géométriques, ces symboles à fonction de rassemblements rituels devaient être accessibles aux dieux des cieux et des montagnes, ainsi que le prouvent les dimensions des tertres et des fossés qui en composent les lignes et les images.

Au fil du temps, beaucoup de ces éléments intermédiaires ont disparu. Il est cependant encore possible de nos jours de visiter quelques uns de ces sites faits images, entre autres par exemple ceux où apparaissent, tracés dans la tourbe, au sud de l'Angleterre, Le géant Cerne Abbas et Le cheval d'Uffington ou, au centre de l'Amérique du Nord, en Ohio et Ontario, les tertres artificiels figurant des serpents; ou encore, outre les pétroglyphes du monde entier (de Peterborough, Ontario, au Sahara), les lignes du Nazca au Pérou.

Le mystère des déserts péruviens n'est toujours pas élucidé. Depuis la redécouverte des lignes et des images du Nazca par Paul Kosok en 1939, on n'a pu, faute d'autres signes laissés par les peuples qui les ont tracées et qui n'ont pas développé une langue communicable, qu'élaborer des conjectures.

Paul Kosok a ainsi émis l'hypothèse du «plus grand livre d'astronomie du monde"; Maria Reiche a montré la grande importance des alignements orientés vers le soleil et les étoiles; Gerald S. Hawkins, dans Beyond Stonehenge n'a pas trouvé de liens remarquables entre les lignes et les éléments cosmiques; Tony Morrison suggère que les lignes représentent les chemins conduisant au sommet des montagnes, sur les lieux de célébration du culte des ancêtres; tout dernièrement, enfin, Johan Reinhard, dans son livre The Nazca Lines (Les lignes du Nazca) démontre de manière convaincante la fonction invocatoire des symboles de fertilité adressés, selon lui, aux dieux des Andes à des fins pratiques: assurer l'alimentation en eau nécessaire à l'irrigation des fermes précolombiennes dans les vallées.

Mes oeuvres (landworks), réalisées entre décembre 1984 et décembre 1986 sur les étendues non archéologiques des déserts, font référence dans quelques cas aux cérémonies de la fertilité, comme le montrent les photos et légendes ci-après. 


\section{COMMENTAIRES}

Le géant Cerne Abbas: symbole de fécondité, comme l'indique le membre pointé vers l'est, d'où vient la chaleur du soleil. La tourbe a été creusée pour exposer la pierre blanche de l'intérieur du paysage. Cette oeuvre représente une tradition qui existait il y a deux mille ans et qui semble avoir été alors présente partout dans le monde.

De la même époque, l'Âge de fer, l'époque des Celtes en Angleterre: un cheval. La même méthode a été utilisée: on a enlevé la tourbe pour montrer le blanc de la terre mère.

Juste à côté du Bouclier canadien, près de Peterborough, les pétroglyphes invoquent la fécondité. Sur le côté droit, une femme. L'oxyde rouge des crevasses représente les menstruations, tandis que des rivières souterraines évoquent les murmures des dieux. Pour les Algonquins de cette région, c'est un lieu magique où il est possible d'entrer en communication avec les esprits des lieux en leur faisant des offrandes destinées à favoriser la fertilité.

Dans le sud de la Californie: Intaglis de Blythe. Représentation d'une femme; le membre mâle a été ajouté récemment.

Aux Etats-Unis, dans le sud-est de l'Ohio, une oeuvre de 400 mètres de long, faite par les Indiens préhistoriques Adena-Hopewell, représentant un serpent en train d'avaler l'oeuf cosmique. Il existe également un autre tertre en forme de serpent, en Ontario, près des pétroglyphes de Peterborough.

Une image des plaines de Nazca, tirée du livre de Maria Reiche, montre les pattes d'un oiseau géant, une frégate, symbole de fertilité pour le désert. Les lignes droites sont alignées avec les points clés de I'horizon au moment des solstices et des équinoxes.

Une image d'une araignée, telle qu'on en voit dans la jungle péruvienne ou brésilienne. Curieusement, le membre reproducteur apparaît comme une patte détachée. Gerald S. Hawkins utilise cet exemple dans son livre Beyond Stonehenge pour montrer que, chez les Indiens Nazca, ces dessins n'étaient pas autre chose que des symboles de fertilité.

Plusieurs représentations ici: encore l'araignée, et un singe et son organe reproducteur. Pour Johan Reinhard, le zigzag qui apparaît au cou de l'oiseau à droite serait le symbole de l'eau, en relation avec les systèmes d'irrigation érigés à côté des rivières par les habitants 
des plaines côtières de Nazca. Ici encore, un singe: les zigzags dans le prolongement de son organe de reproduction transposent sous forme géométrique les systèmes d'irrigation des plaines.

Une image des Andes: la ville de Pisac, près de Cuzco. Les Incas, qui n'ont pas fabriqué d'images sur les plaines, vénéraient plutôt les dieux du soleil. Sur le Nazca, ils invoquaient les dieux des montagnes pour obtenir de l'eau dans les vallées, comme le montre ici la présence d'un colibri.

À propos de cette image-ci qui représente peut-être un oiseau, Tony Morrison avance, dans Pathways to the gods (Les chemins aux dieux) que ces lignes constitueraient des chemins de pélerinage destinés à la célébration des ancêtres, tandis que pour Johan Reinhard dans The Nazca Lines, ils serviraient à aller invoquer les dieux des pluies.

Sur ces images du versant du Pacifique, une sorte d'hybride de condor et de baleine destiné à invoquer les dieux de la pluie.

Enfin, un dernier oiseau, découvert en 1983, alors que les précédentes découvertes, faites par Paul Kosok puis Maria Reiche, datent des années 40 et 50 . D'après sa facture de tête de trophée il proviendrait peut-être de la culture des Paracas, antérieure à celle des Nazcas.

Ceci est une de mes premières oeuvres, datant de décembre 1984, réalisée au Pérou sur les plaines de Nazca. C'est une prolongation, dans un contexte contemporain, des tendances antérieures. Il s'agit d'une momie en position foetale, entourée d'un cordon ombilical, symbole d'éternité. D'après Johan Reinhard, les anciens auraient utilisés les momies à titre d'intercesseurs auprès des dieux en vue d'obtenir de l'eau.

Les dimensions des images que j'ai fabriquées dans cette région sont de l'ordre de 100 mètres $x 100$ mètres. Elles se situent dans des zones non archéologiques, cela afin de ne pas abîmer les images qui sont déjà là, au nord de Nazca, entre les villes de Nazca et Palpa. Ici une vue au sol, au sud de Nazca.

«Una cabeza trofeo» ou une tête de trophée, que j'ai faite d'après une poterie du Musée archéologique de Lima. La bouche est pincée avec des tiges. Les ouvriers et moi avons balayé ou creusé à une profondeur de 5 à 10 pouces, sur une largeur d'un pied et demi, pour faire apparaître le contraste entre le sable blanc, plus fin d'en 
dessous et le sable gris foncé, moins granuleux de la surface. Au Québec ou au Canada, cette oeuvre ne durerait pas plus de quelques jours ou quelques mois, mais dans cette région privée de pluie elle peut durer très longtemps. Les autres images qu'on voit ici proviennent d'une période allant du Ve siècle avant notre ère au XVe siècle de notre ère.

Voici maintenant un projet intitulé Antipodes Cambodge, dans lequel je voulais représenter le point exactement opposé de notre planète. Voici donc une carte inversée de ce pays. Une autre oeuvre "mondiale», dans la lignée de celles que j'ai déjà faites, comme La ligne mondiale, le projet Contacts ou La lacune des trois miles. La première de cette nouvelle série s'intitule Antipodes.

Ici c'est une spirale dont le titre est $\mathcal{E} \mathrm{El}$ remolino Cela ressemble à un point d'interrogation. Je voulais représenter ces tourbillons de sable qu'on voit souvent sur les plaines. Là c'est une ligne qui vient des montagnes et forme une spirale en tournant sur elle-même.

En décembre 1985, j'ai voulu montrer un symbole d'eau sur cette surface très sèche, inanimée. L'image qui en a résulté est $\mathrm{El}$ pulpo (la pieuvre). Pour se faire une idée à l'échelle de cette vue aérienne, il faut regarder ici, dans cette image prise de près, les traces de roues ou des chevaux ainsi que les gens ici et là. On voit comment le vent a déjà commencé d'effacer l'oeuvre.

Cette oeuvre s'intitule El dios de los aires (le dieu des vents). C'est l'image que les Nazcas employaient pour montrer ce dieu. Je l'ai trouvée sur quelques poteries dans un musée d'lca, une ville entre Lima et Nazca. Même si le vent qui a commencé à effacer les sections blanches de l'image rend l'ensemble gris comme le reste du désert, il restera les creux à la surface. (C'est grâce à ces creux que Kosok a fait ses découvertes en 1939, creux que Reiche, lors des recherches entreprises à partir de 1946, a nettoyés pour rétablir les contrastes de ton.) Un dieu en forme de zigzag, une combinaison de styles. La tête un peu dans le style des Paracas. En creux, entouré de pierres. De près: l'avion dans lequel nous sommes pour prendre les photographies est peut-être à cinquante pieds au-dessus de cette image.

Sur la route panaméricaine, tout près d'Arequipa, à la frontière: une autre spirale, mais de forme rectangulaire. Puis une perspective autre de Nazca fret. A Chavina, à défaut de pelles nous avons utilisé nos pieds - une méthode comparable à celle des empreintes, que j'ai utilisée ici au Québec pour des oeuvres dans la neige. 
A Huarez, dans les Andes, en décembre 1986. Je voulais faire une forme objective/positive, La boca (la bouche) d'un puma. On peut voir les dents de ce dieu, c'est-à-dire les pierres placées sur la rive de cette rivière.

Entre cet endroit et Nazca, nous avons fabriqué une autre image de pluie. Ici on voit un nuage avec des éclairs et la pluie formant lacs et rivières. La taille moyenne de ces images, ne l'oublions pas, est de 100 mètres de diamètre.

Voici le moyen d'exorciser la peur de l'écrasement de l'avion: un dessin d'avion écrasé sur le désert!

Maintenant, une autre momie, (\# 2), sous divers angles. On distingue le visage du côté gauche, et les pieds et les jambes du côté droit.

Sur un terrain privé appartenant à Jose Barahona. Vue d'un Huari pensant aux systèmes d'irrigation, comme l'indiquent les zigzags. J'ai trouvé cette image sur quelques pots au Musée archéologique de Lima.

L'oasis de Baharona. Voici La momia número tres (la momie \# 3) dans son linge de mort. J'ai trouvé cette momie dans un musée d'Arequipa. Les lignes qui l'entourent constituent le barda (bundle, en anglais) du mort, sur lequel le zigzag, toujours le zigzag, symbolise la fertilité dans l'autre vie, dans l'autre dimension.

\section{Voilà.}

\section{Ouvrages cités}

Exhibition catalogue: Peruvian Ground Drawings, Kunstraum München, Münich 1974.

Hawkins, Gerald S.: Beyond Stonehenge, Harper E Row, New York, 1973.

Kosok, Paul: The Mysterious Markings of Nazca, Natural History, LV, 1947.

Morrison, Tony: Pathways to the Gods, London, Paladin, 1980.

Reiche, Maria: Mystery on the Desert, Heinrich Fink Gmbh $\&$ Co., Stuttgart, West Germany, 4th. edition, 1982.

Reinhard, Johan: The Mazca Lines, Editorial Los Pinos E.I.R.L., Lima, Pérou, 1985. 


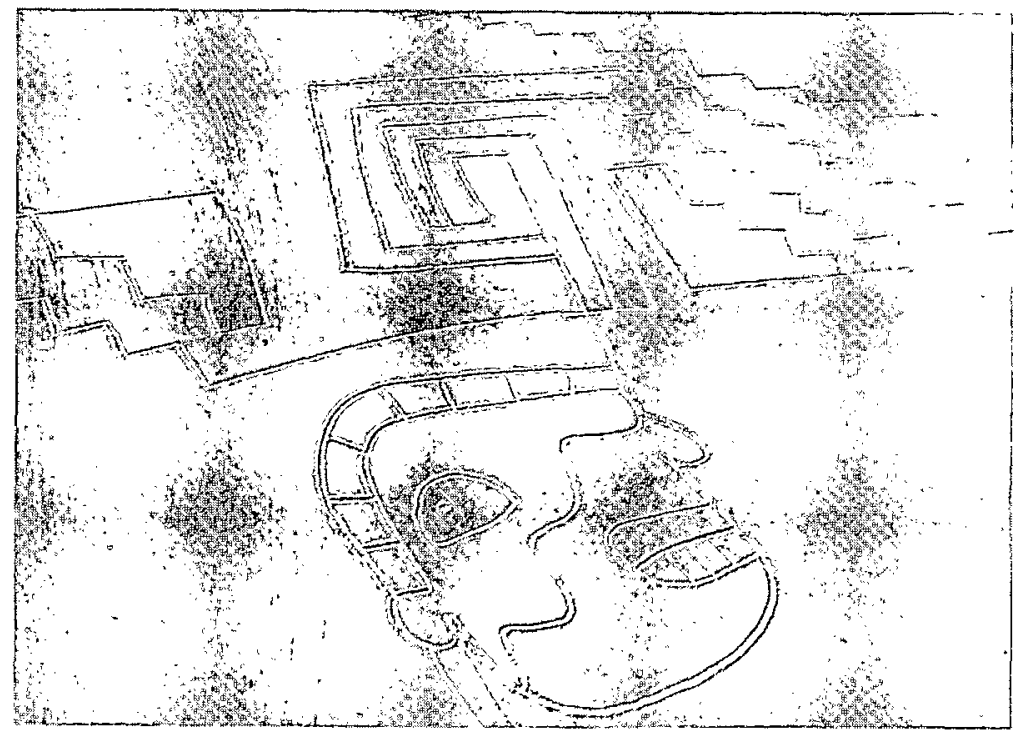

Huari, Imigation plan, Décembre 1986. 100x130m

Grattage du sable de surface (réseaux de canaux)

Oasis Barahona, Mazca, Pérou.

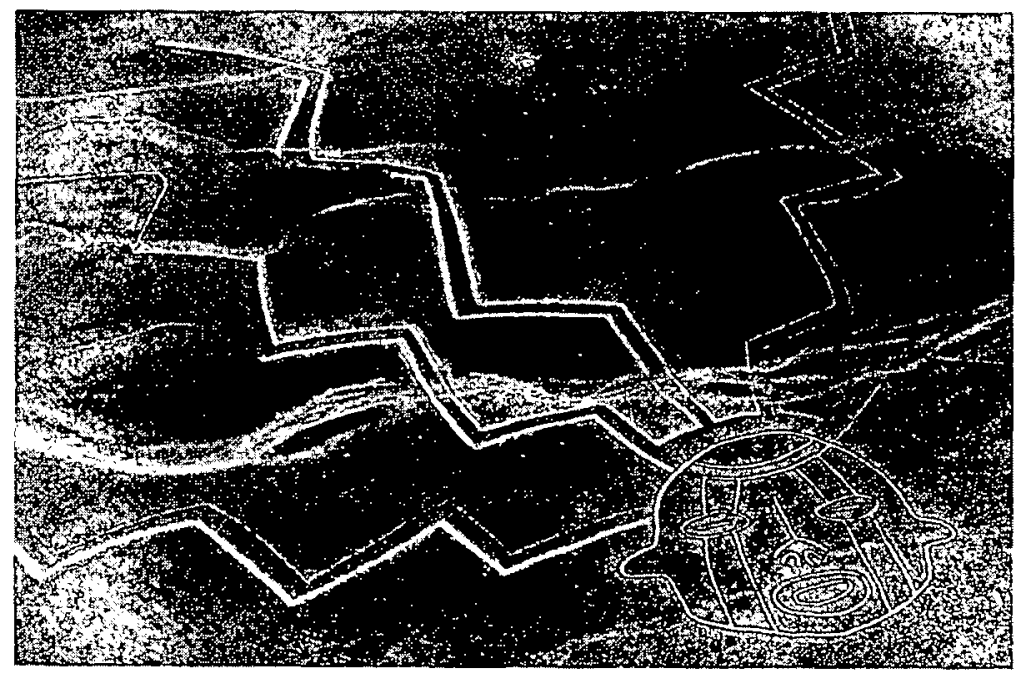

Zig Zag God, novembre 1985, 100x100m

Grattage du sable de surface avec mise en place de pierres aux bords (l'eau qui dévale de la Cordillère des Andes)

Nazca, Pérou. 


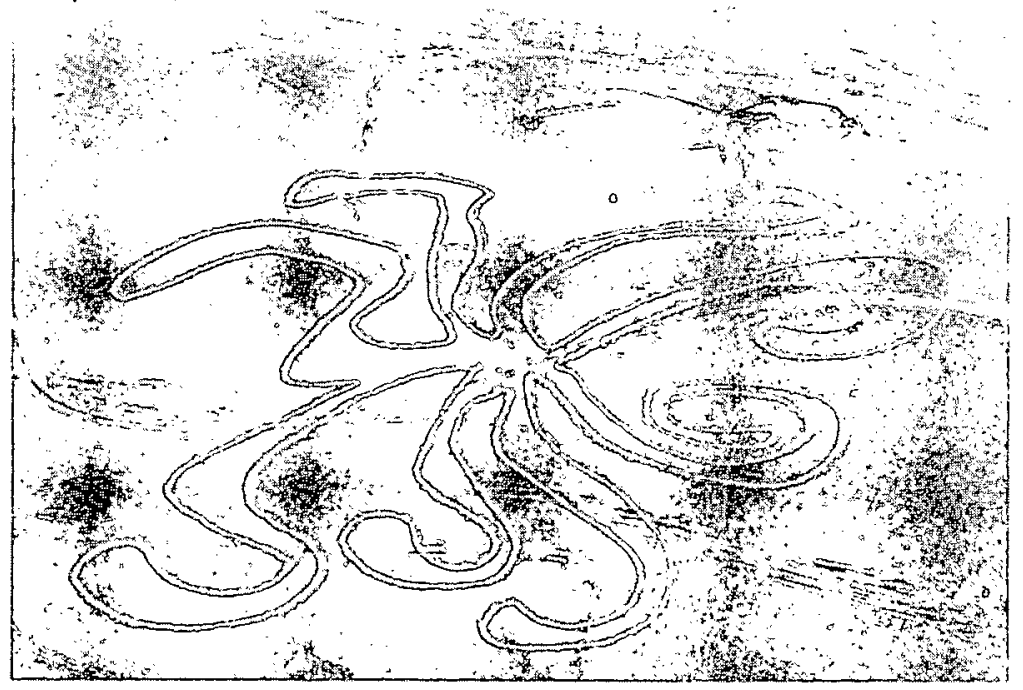

El pulpo, novembre $1985,100 \times 100 \mathrm{~m}$

Grattage du sable de surface; image d'une pieuvre sur le désert côtier (avec piste d'autos et camions)

Nazca, Pérou.

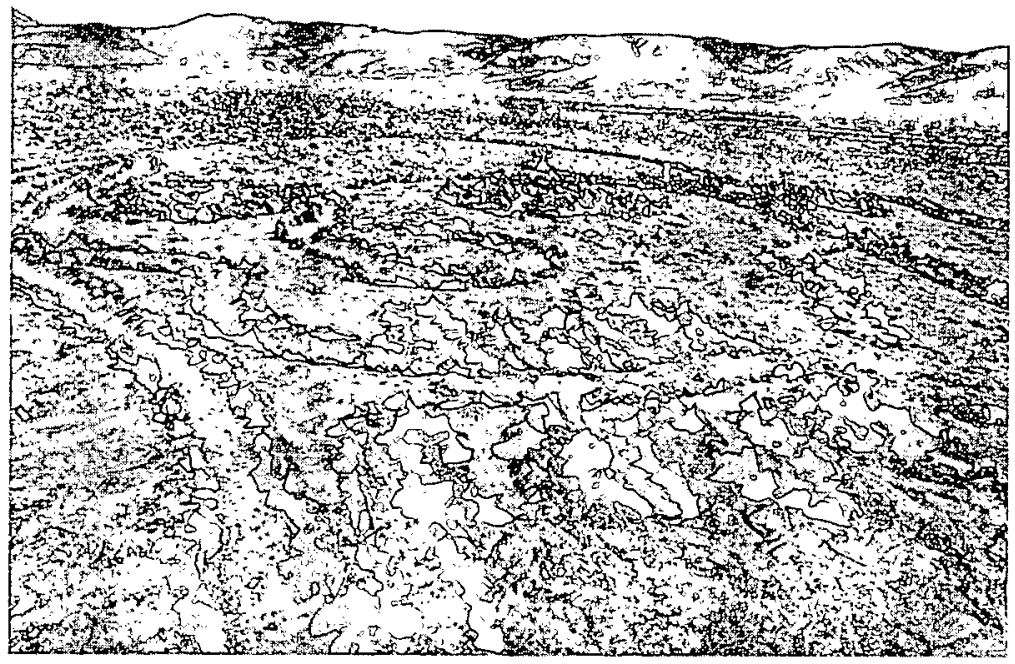

Momia no. 2, novembre $1986,100 \times 100 \mathrm{~m}$

Grattage du sable de surface avec mise en place de pierres aux bords (vue du sol) Nazca, Pérou. 


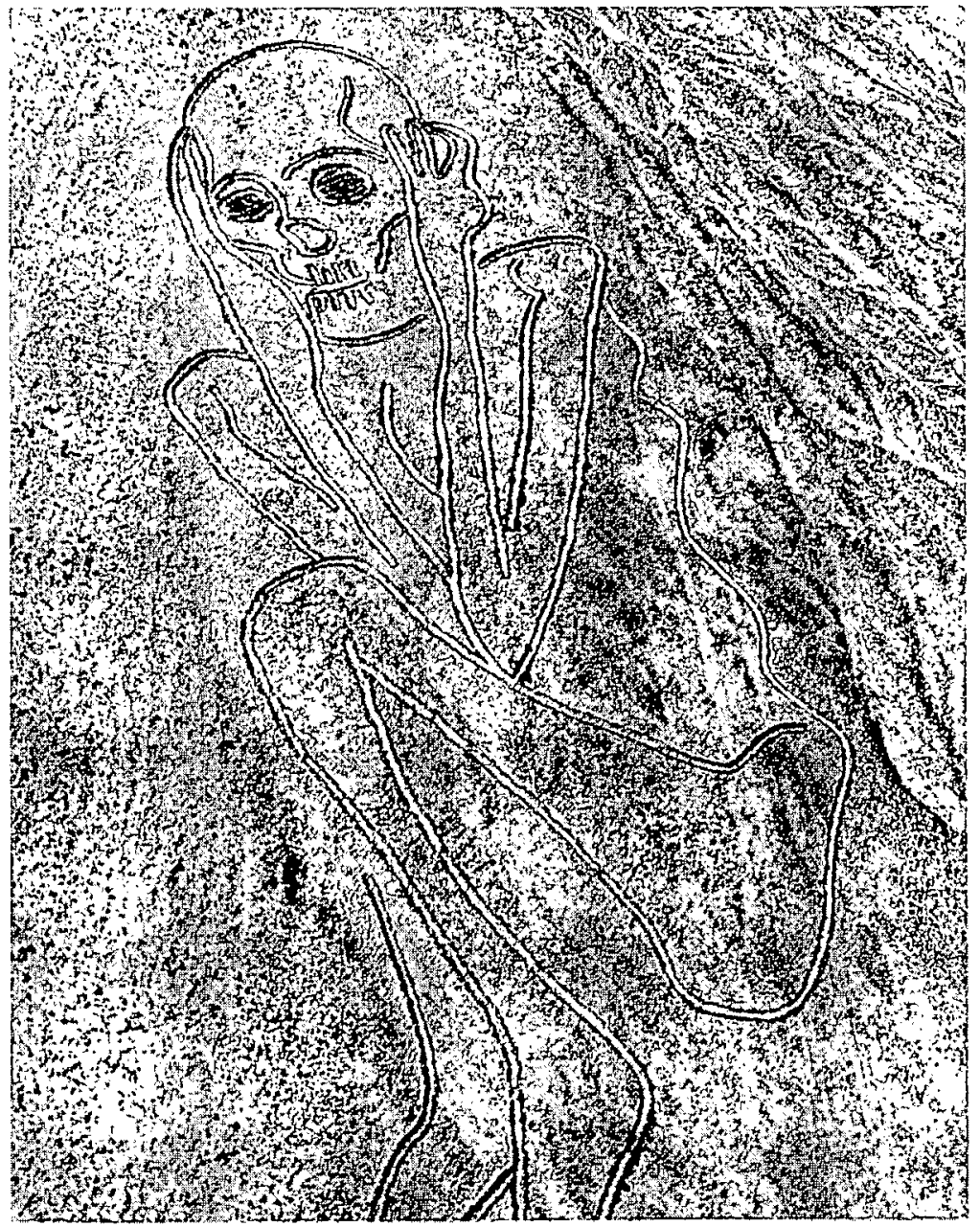

Momia, no. 2, novembre 1986, $100 \times 100 \mathrm{~m}$

Grattage du sable de surface avec mise en place de pierres aux bords (vue aérienne) 


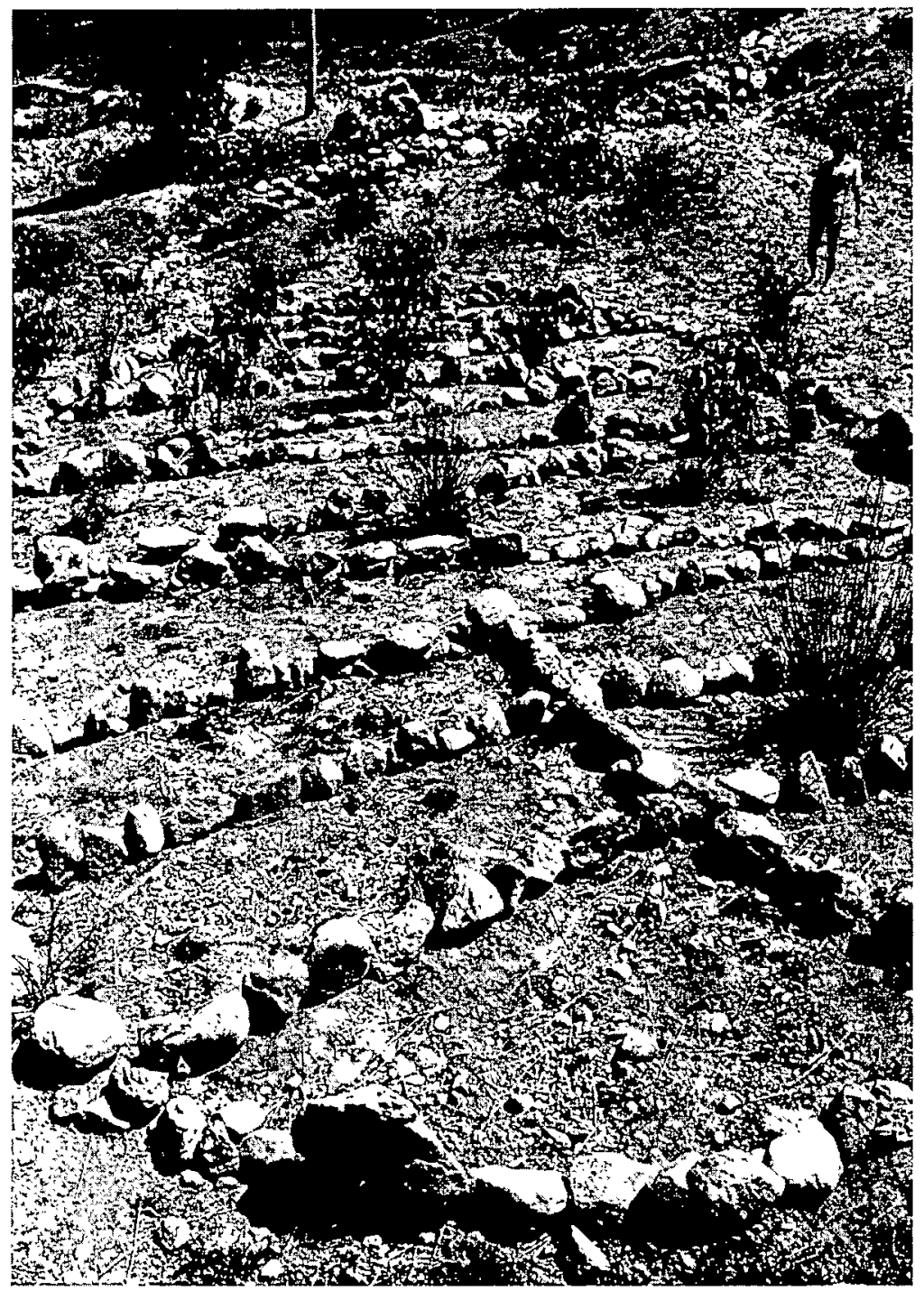

La boca, novembre $1986,10 \times 20 \mathrm{~m}$

Placement des pierres au côté d'une rivière andienne (la bouche d'un félin volant des Andes)

Huaraz, Pérou. 


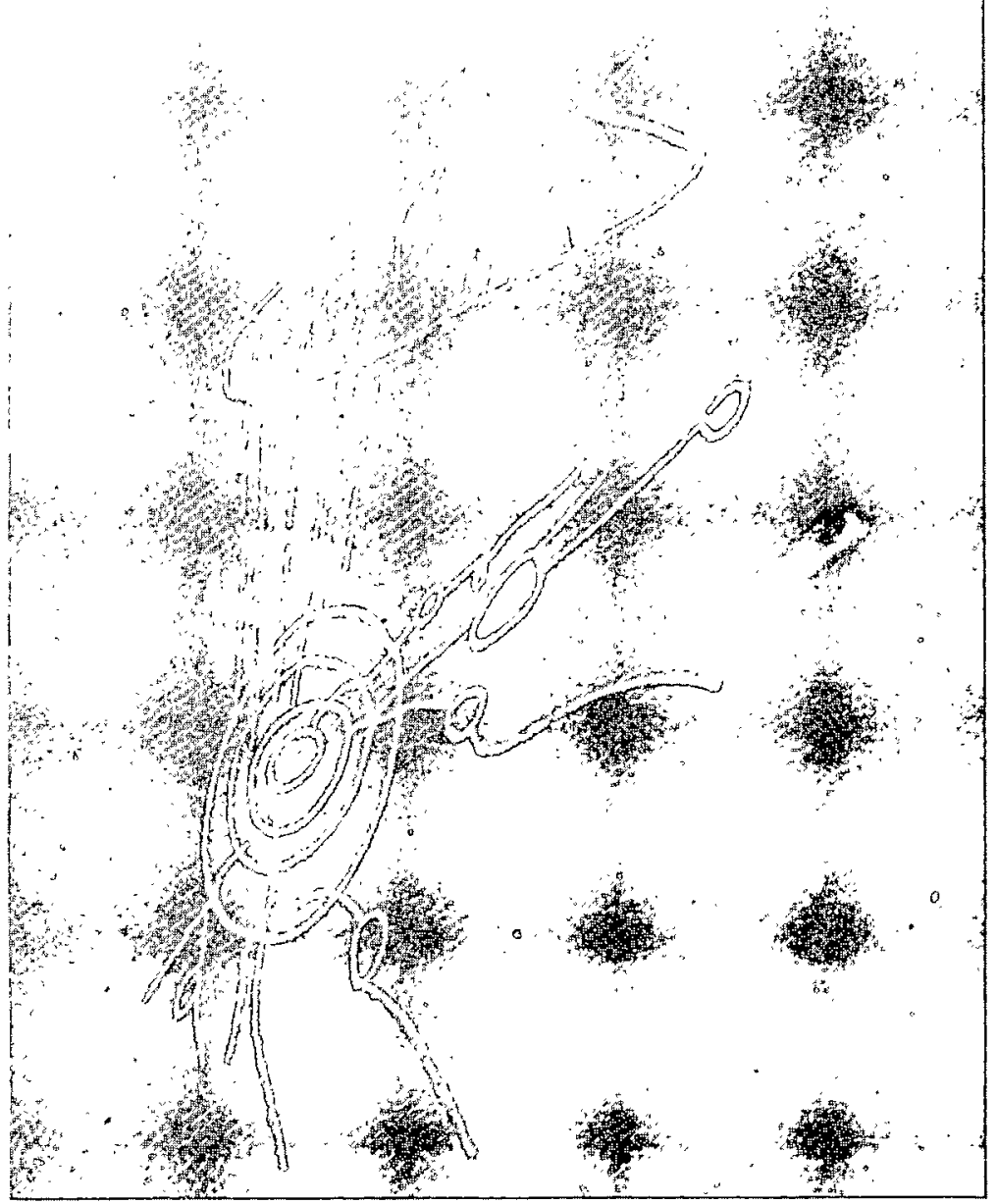

La pluie, novembre $1986,100 \times 100 \mathrm{~m}$

Grattage du sable de surface (nuage, éclairs, pluies, rivières, lacs, destinés au désert) Nazca, Pérou. 